\title{
Multisite Field Potential Recordings and Analysis of the Impulse Propagation Pattern in Cardiac Cells Culture
}

\author{
S Jacquir ${ }^{1}$, S Binczak ${ }^{1}$, M Rossé ${ }^{1}$, \\ D Vandroux $^{2}$, G Laurent ${ }^{3}$ P Athias $^{2}$, JM Bilbault $^{1}$ \\ ${ }^{1}$ Laboratory LE2I UMR CNRS 5158, University of Burgundy, Dijon, France \\ ${ }^{2}$ Institute of Cardiovascular Research, University Hospital Center, Dijon, France \\ ${ }^{3}$ Cardiology Department, University Hospital Center, Dijon, France
}

\begin{abstract}
To provide further insights into the impulse propagation between cardiac myocytes, we performed multiparametric studies of excitation spread with cellular resolution in confluent monolayers of cultured cardiomyocytes (CM). Simultaneous paired intracellular recordings of action potentials in two individual CM revealed slight periodic spontaneous advances/delays in the interspike time lag. Multisite field potential recordings performed with microelectrode arrays (MEA) confirmed random and iterative cycle-to-cycle changes in the direction of excitation spread. These local spontaneous variations in the cardiac impulse propagation pathways may be a safety process protecting against microscopically discontinuous conduction, and abnormality of this natural process could contribute to the genesis of some heart arrhythmias.
\end{abstract}

\section{Introduction}

The cardiac conduction system, which enables fast and coordinated contraction of the heart, relies on specialized intercellular permeable junction channels, named gap junction $[1,2]$. Conduction abnormalities may occur either from changes in the action potential contour or from electrical uncoupling at gap junctions, or both [3, 4]. However, the detailed electrophysiological understanding of this arrhythmic propensity is still unclear, because of the lack of experimental models allowing routine simultaneous monitoring of action potentials and of cell-cell electrical coupling. Experimental model are thus mandatory to get relevant insights into this kind of arrhythmogenesis. We confirmed here that cardiac muscle cells cultivated from newborn rat hearts display differentiated functional properties, and therefore represent a valuable cellular model of the myocardial excitability, as demonstrated earlier $[5,6]$.
To provide further insights into the impulse propagation between cardiac myocytes, we performed multiparametric studies of excitation spread in confluent monolayers of cultured cardiomyocytes (CM) using either simultaneous paired intracellular microelectrodes or microelectrode arrays (MEA). The section 2 describe the materials and methods used in our study, the section 3 shows some results and then the last section 4 is devoted to the conclusion and discussion.

\section{Materials and methods}

\subsection{Cardiomyocyte culture preparation}

Neonatal ventricular myocytes were prepared from 1 to 4 days-old Wistar rats by trypsin-based enzymatic dispersion as described previously [7]. The cell suspension was preplated twice in Ham's F10 medium supplemented with fetal calf serum (FCS) and penicillin/streptomycin $(100 \mathrm{U} / \mathrm{ml})$ in order to increase cardiomyocyte proportion. Cardiomyocyte-rich cultures $(>90 \%)$ were seeded at a final density of $10^{5}$ cells per $\mathrm{cm}^{2}$ in supplemented Ham's $F 10$ medium. Cultures were incubated in a humidified incubator $\left(95 \%\right.$ air, $5 \% \mathrm{CO}_{2}$ at $\left.37^{\circ} \mathrm{C}\right)$ and were used after 4-5 days of growth, a step at which confluent and spontaneously beating cell monolayers were obtained.

\subsection{Paired intracellular recordings}

For intracellular electrophysiology, cardiomyocytes were plated onto $60 \mathrm{~mm}$ tissue culture dishes. CM cultures were mounted in a thermo-controlled $\left(37^{\circ} \mathrm{C}\right)$ bath on an inverted microscope as previously described [5]. Simultaneous paired intracellular recordings of action potentials in two individual CM spaced 100-300 $\mu \mathrm{m}$ apart were done using two independently driven conventional glass microelectrodes. The contractions were simultaneously recorded 
by the use of a video cell motion detector [6]. The electromechanical signals were acquired, stored and analyzed as reported previously [5].

\subsection{Multielectrode array recordings}

CM were grown on multi-electrode arrays (MEA) allowing non-invasive synchronous multifocal field potential (FP) recordings. The MEA consists of 60 substrateintegrated microelectrode arrays $(8 \times 8$ matrix, $30 \mu \mathrm{m}$ electrode diameter, $200 \mu \mathrm{m}$ inter-electrode distance). Data were acquired and analyzed with a customized platform programmed with MATLAB (Mathworks) in order to provide two-dimensional electrophysiological maps derived from these multisite FP recordings.

\section{Results}

\subsection{Paired intracellular recordings}

The transmembrane voltages recorded in cultured cardiomyocyte pairs were characterized by high amplitude, fast rising action potentials (Fig. 1), as previously described. This observation confirmed that these cells display differentiated functions that were very similar to those of mature cardiac muscle cells [5]. The action potentials were regularly coupled to synchronous cell shortening, with a highly constant time delay between the action potential and the contraction. The time delay between the

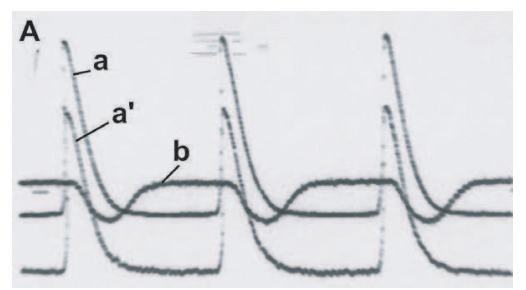

Figure 1. Slow sweep speed display of microelectrode pair recording of action potentials (a and a') and of contraction (b) from two rat cardiac muscle cells (intercellular distance: $200 \mu \mathrm{m}$ ).

two initial voltage upstrokes (Fig. 2), allowed the calculation of the propagation velocity between the two individual cardiomyocytes. In these conditions, the mean conduction velocity was $30.4 \pm 2.2 \mathrm{~cm} / \mathrm{s}$ (mean $\pm \mathrm{SD}$ ). Whereas we observed highly stable action potential contours and interbeat periods, we also noticed slight periodic spontaneous advances/delays in the interspike time lag $(2.2 \pm 1.1 \mathrm{~ms}$, $6.4-0.2 \mathrm{~ms}$ range) (Fig. 3). We checked that these propagation instabilities were independent on the rising speed of the action potential upstroke [8], which is a key determinant of the conduction velocity. This phenomenon may

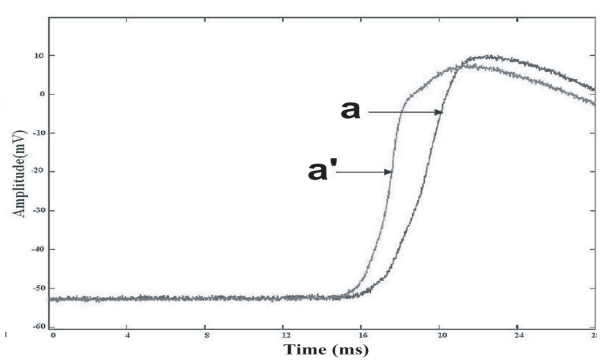

Figure 2. Same than Fig.1 at fast sweep speed, showing initial voltage upstroke of two superimposed action potentials (a and a').

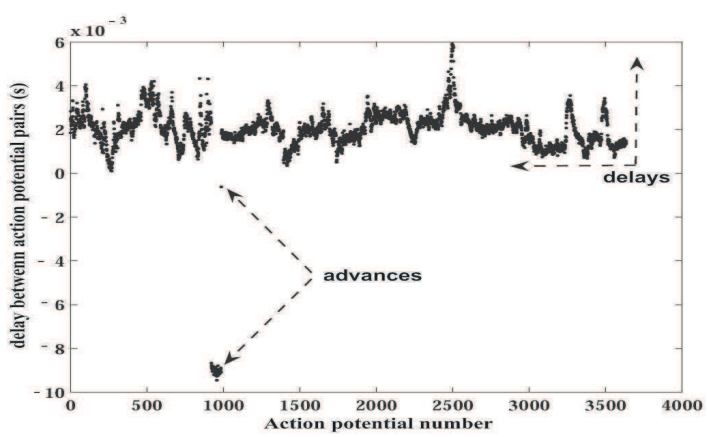

Figure 3. Time-related spontaneous fluctuations in delay between action potentials of cardiomyocyte pair simultaneously recorded with intracellular microelectrodes

be caused by natural fluctuations in cell-to-cell electrical coupling and/or in conduction pathway.

\subsection{Multielectrode array recordings}

Multielectrode arrays (MEA) offer the opportunity to obtain information about the electrical interactions within a larger population of cardiomyocytes. Each of the $60 \mathrm{mi}-$ croelectrodes embedded in the bottom of MEA dishes allow the long-term recordings of the extracellular electrical activity (field potentials, FP), i.e., the cellular ECG, which reflects the local changes of the membrane potentials (Fig. 4). Briefly, the maximal FP amplitude (Fig. 4) reflected the action potential upstroke velocity (Fig. 1), the FP duration was an accurate measure of the action potential duration and the time delay between FP allowed precise rate measurements. The data acquired from the MEA were used to construct two-dimensional, color-coded maps showing the spatial and temporal distribution of amplitude and time parameters calculated from the extracellular electrograms.

Figure 5 displays representative spatial distributions of FP time parameters from a monolayer culture of ventricular cardiomyocytes. These data show that the duration and the spontaneous periodicity of the cellular ECG complex 


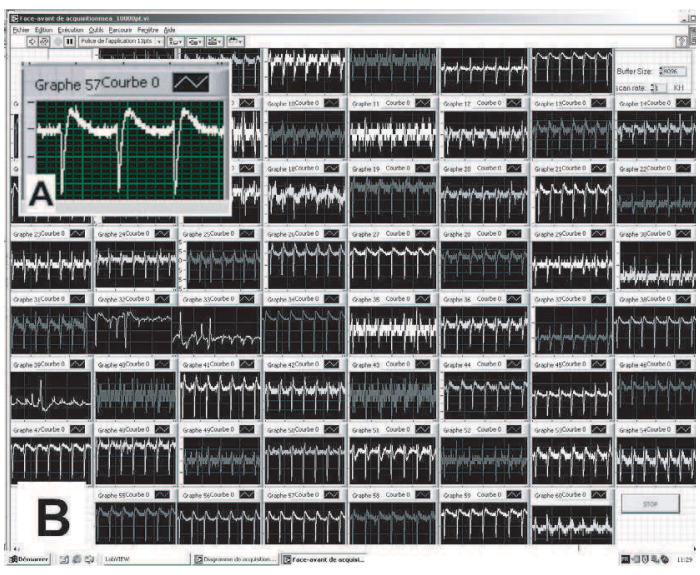

Figure 4. (A, inset). Single Cardiomyocyte ECG (field potential) recorded in MEA by one of the 60 substrateintegrated extracellular electrodes. (B). Simultaneous display of the field potentials recorded in MEA by each of the 60 substrate-integrated extracellular electrodes.

were homogeneously distributed throughout the recording area. These field potential mapping confirmed thus that the cultured cardiomyocytes displayed synchronized electrical activity and that the FP-derived parameters remained stable and homogeneous, which has already been described for conventional endocellular recordings [5].

The conduction velocity was calculated from the time delay between isochronal FP (local activation time; LAT) recorded by the different pairs of extracellular microelectrodes, one of these electrodes being arbitrarily taken as the time reference. To assess impulse propagation, these data were then used to create activation maps, from which direction and velocity of action potential propagation can be appreciated.

The figure 6 shows typical activation pattern in control conditions derived from the cellular ECG recorded in the same MEA culture dish at a two cycles interval. The second map (b) being built two complete cell depolarization lags after the first map (a). Between the two maps, there was a shift in the activation pattern of the wave-front, corresponding to spontaneous fluctuations in the propagation pathways within the two-dimensional cell sheet. In addition, this observation was consistent with the data obtained using the intracellular microelectrode pairs.

\section{Discussion and conclusions}

In this study, we first investigated the electrical conduction with the use of intracellular microelectrode pairs. This endocellular method is the gold standard for the investigation of intercellular electro tonic conduction, because the action potential upstroke, which governs the conduc-

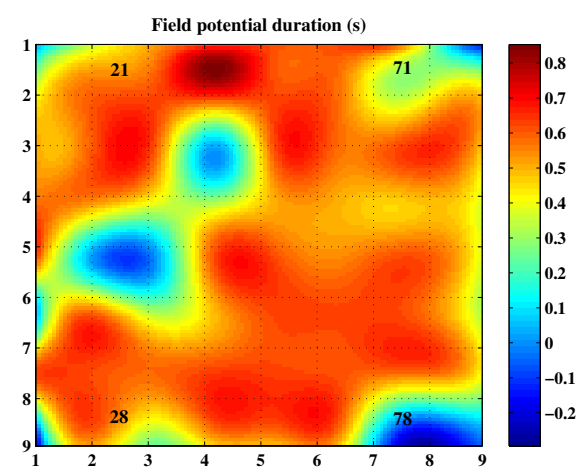

(a)

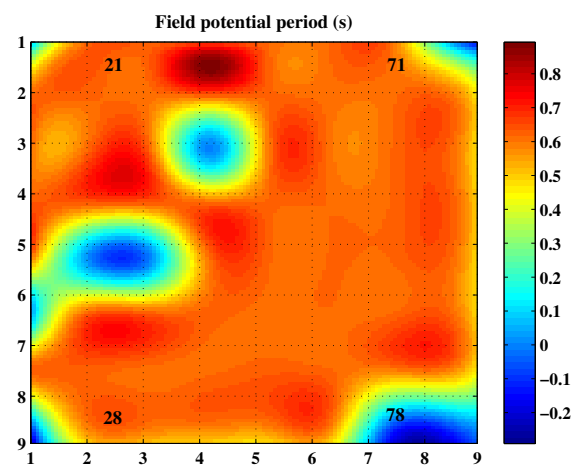

(b)

Figure 5. Spatial distribution map of field potentials obtained from cardiomyocytes after 6 day growth on MEA. (a): ECG duration mapping (b): periodicity mapping. Data were smoothed by interpolation processing. In the two maps, the central left contrasted spots correspond to failed cell-electrode coupling.

tion speed, can be simultaneously monitored. Using this method, we calculated the conduction velocity at about $30 \mathrm{~cm} / \mathrm{s}$, which was similar to the conduction velocity in ex vivo preparation and consistent with myocardial tissue [9]. This paired microelectrode investigation revealed for the first time unstable intercellular activation delays suggesting spontaneous variations in cell coupling and/or local meandering conduction among cardiomyocytes. This apparent stochastic conduction process appears to be unrelated to other action potential parameters, in particular to the upstroke velocity. To confirm our hypothesis in a larger scale, we thereafter explored the cardiomyocyte cultures in MEA. This device appeared as a convenient and accurate tool for a two dimension delineation of the cardiac conduction process at the cellular level, as emphasized previously [10]. This MEA analysis showed again stable spontaneous pacemaking activity with synchronous FP trains. Moreover, these measurements showed a rather 


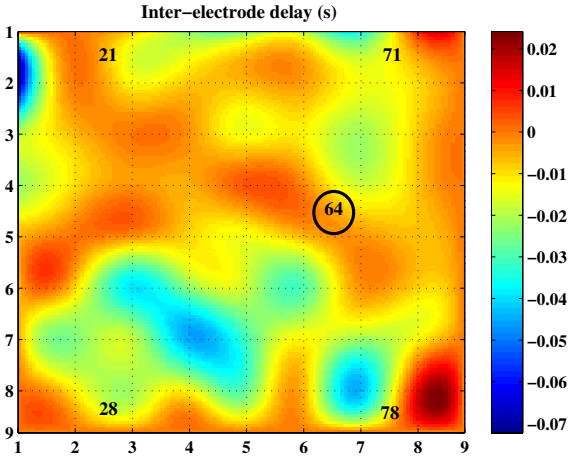

(a)

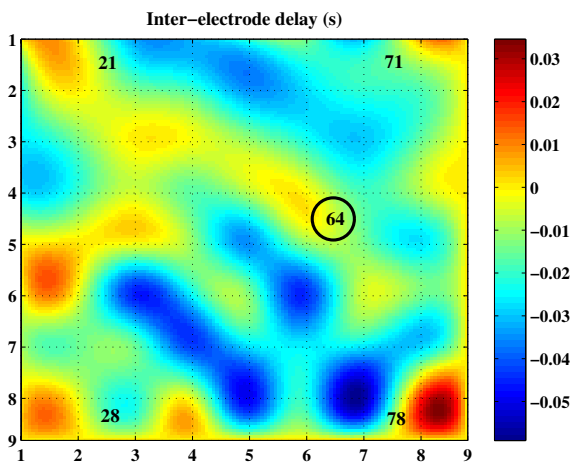

(b)

Figure 6. Activation map constructed by two dimensional plotting of the local activation time measured from cardiomyocytes after 6 day growth on MEA. (a): reference activation map (cycle $n$ ). (b): activation map constructed 2 period latter $($ cycle $n+2)$. The time reference electrode (circled) is the same. In the two maps, the central left contrasted spots correspond to failed cell-electrode coupling.

homogenous spread of excitation throughout the recording area, with slower conduction spots corresponding assumingly to nonmyocytes or lower myocyte density patches. The observation of this cell density-dependent local variation in the FP propagation was consistent with previous report [11], and may mimic electrical wave propagations around anatomical obstacles in the heart [9]. The relevant new finding was that the direction of the excitation spread was affected by spontaneous and rapid changes, within the interspike time delay range. This finding made at a multicellular level confirmed our initial hypothesis based on observations made with the paired microelectrode model. To our knowledge, there is no comparative data in the literature. Stable propagation patterns have been oppositely reported [12]. The sole changes in conduction velocity and direction which have been hitherto described have been linked to developmental stages of the cell cultures [12] or to the pacing rate [13]. To conclude, we demonstrated in the present study that the propagation pattern in cardiac myocyte culture was affected by random and iterative cycle-to-cycle changes that may be related to gap junction functions. These spontaneous fluctuations of the activation pathways may represent local adjustment mechanisms to get around microscopical areas of conduction block. It might be foreseen that disturbance of this natural safety process could lead to cardiac arrhythmias.

\section{References}

[1] Moorman AFM, Christoffels VM, Anderson RH. Anatomic substrates for cardiac conduction. Heart Rhythm. Aug 2005; 2: 875886.

[2] Rohr S. Role of gap junctions in the propagation of the cardiac action potential. Cardiovasc Res 2004; 62: 309-322.

[3] Jongsma HJ, Wilders R. Gap junctions in cardiovascular disease. Circulation Research 2000; 86: 1193-1197.

[4] Smits JPP, Veldkamp MW, Wilde AAM. Mechanisms of inherited cardiac conduction disease. Europace . March 2005; 7: 122-137.

[5] Tissier C, Bes S, Vandroux D, Fantini E, Rochette L, Athias P. Specific electromechanical responses of cardiomyocytes to individual and combined components of ischemia. Can. J. Physiol. Pharmacol., 2002;80:1145-1157.

[6] Athias P, Grynberg A (1987) Electrophysiological studies on heart cells in culture. In: The heart cells in culture (ed. Pinson A), pp. 125-158. CRC Press, Boca Raton, Florida.

[7] Grynberg A. Primary rat cardiac cell culture: diet of the mother rats as a determinant parameter of cardiomyoblast production from neonates. Biol. Cell 1986; 57: 89-92.

[8] Jacquir S, Laurent G, Tissier C, Vandroux D, Devillard L, Brochot A, Rochette L, Binczak S, Wolf JE, Bilbault JM, Athias P. Simultaneous paired intracellular microelectrode study of the impulse propagation in cardiomyocyte culture. Archives des Maladies du Coeur et des Vaisseaux, 2005;98(4):392.

[9] Pillekamp F, Reppel M, Brockmeier K, Hescheler J. Impulse propagation in late-stage embryonic and neonatal murine ventricular slices. J Electrocardiol 2006; 39: 425 e421-424.

[10] Stett A, Egert U, Guenther E, Hofmann F, Meyer T, Nisch W, Haemmerle H. Biological application of microelectrode arrays in drug discovery and basic research. Anal. Bioanal. Chem., 2003;377:486-495.

[11] Banach K, Halbach MD, Hu P, Hescheler J, Egert U. Develpement of electrical activity in cardiac myocyte aggregates derived from mouse embryonic stem cells. Am. J. Physiol. Heart Circ. Physiol., 2003;284:H2114-23.

[12] Hescheler J, Halbach M, Egert U, Lu ZJ, Bohlen H, Fleischmann BK, Reppel M. Determination of electrical properties of ES cellderived cardiomyocytes using MEAs. J Electrocardiol 2004; 37 Suppl: 110-116.

[13] Kondratyev AA, Ponard JGC, Munteanu A, Rohr S, Kucera JP. Dynamic changes of cardiac conduction during rapid pacing. American Journal Of Physiology Heart And Circulatory Physiology. Apr 2007; 292: H1796-H1811.

Address for correspondence:

\section{Dr Sabir JACQUIR}

Laboratory of Electronics, Computer Science and Image (LE2i), University of Burgundy, 9 avenue Alain Savary, BP 47870,

21078 Dijon, France,

sjacquir@u-bourgogne.fr 